\section{Cahiers de Narratologie}

Analyse et théorie narratives

$31 \mid 2016$

Sérialité narrative. Enjeux esthétiques et économiques

\title{
Le cliffhanger : un révélateur des fonctions du récit mimétique
}

Raphaël Baroni*

\section{(2) OpenEdition}

\section{Journals}

\section{Electronic version}

URL: http://journals.openedition.org/narratologie/7570

DOI: $10.4000 /$ narratologie. 7570

ISSN: $1765-307 X$

Publisher

LIRCES

\section{Electronic reference}

Raphaël Baroni*, «Le cliffhanger : un révélateur des fonctions du récit mimétique », Cahiers de Narratologie [Online], 31 | 2016, Online since 22 December 2016, connection on 19 April 2019. URL : http://journals.openedition.org/narratologie/7570; DOI : 10.4000/narratologie.7570

This text was automatically generated on 19 April 2019

Article L.111-1 du Code de la propriété intellectuelle. 


\title{
Le cliffhanger : un révélateur des fonctions du récit mimétique
}

\author{
Raphaël Baroni*
}

\section{AUTHOR'S NOTE}

*Je remercie en particulier Anaïs Goudmand et Luke Terlaak Poot pour leur relecture et leurs suggestions.

\section{Suspendu au bord d'une falaise}

1 Ainsi que l'affirme David Lodge, «il pourrait difficilement y avoir une situation plus productive de suspense que celle d'un homme accroché par le bout de ses doigts au bord d'une falaise, incapable de grimper pour se mettre en sécurité, d'où le terme “cliffhanger" » (Lodge $2011:$ 13, m.t. ${ }^{1}$.). Lodge suggère que l'on doit l'origine de ce terme à un auteur du XIX ${ }^{\mathrm{e}}$ siècle, Thomas Hardy, qui dans son roman A Pair of Blue Eyes (Les Yeux bleus, 1873), aurait imaginé une scène dont la tension dépend d'une situation narrative jusqu'alors inédite :

À peu près au milieu de Les Yeux Bleus, la jeune et relativement frivole héroïne, Elfridge, fille d'un vicaire de la Cornouaille, emporte avec elle un télescope au sommet d'une haute falaise dominant le canal de Bristol, dans le but de guetter un navire qui, revenant d'Inde, ramène à son bord le jeune architecte avec lequel elle s'est fiancée en secret. Elle est accompagnée par Henry Knight, un ami de sa bellemère, un homme d'âge mûr et un intellectuel qui s'est déclaré auprès d'elle et pour lequel elle est en train de ressentir une attraction coupable. Alors qu'ils sont assis au sommet de la falaise, le chapeau de Knight s'envole vers le rebord et, quand il tente de le récupérer, il se trouve dans l'impossibilité de remonter la pente glissante, ce qui se termine par une chute à pic de plusieurs centaines de pieds. Les efforts impétueux d'Elfridge pour l'aider ne font qu'empirer les choses et, alors qu'elle grimpe vers la sécurité, elle le fait glisser par inadvertance, l'amenant 
encore plus proche de la catastrophe. "Alors qu'il était lentement en train de glisser pouce par pouce [...] Knight eut un dernier élan désespéré pour attraper la plus basse touffe d'herbe - la dernière trace de végétation affamée située à l'endroit où la pierre apparaissait dans toute sa nudité. Elle arrêta sa chute. Maintenant, Knight était littéralement suspendu par les bras. » [...]

Elfridge disparaît de la vue de Knight, probablement pour aller chercher des secours, bien qu'il sache qu'ils se trouvent éloignés de plusieurs miles de l'habitation humaine la plus proche. Qu'arrive-t-il après ? Knight survivra-t-il ? Et si c'est le cas, comment? Le suspense ne peut être durable qu'en repoussant les réponses à ces questions $^{2}$. (Lodge 2011 : 13-14, m.t.)

Dans ce compte rendu, Lodge souligne le lien qui peut être établi entre le suspense, la suspension du récit (voire la "suspension » du personnage) et le cliffhanger, mais en réalité, il est important de réaliser qu'il s'agit de trois phénomènes relativement autonomes. Premièrement, le cliffhanger se distingue du suspense (et également de ce pic de tension que l'on désigne comme le climax) par la nature du délai introduit entre les questions et les réponses qui structurent l'intrigue. En effet, pour qu'il y ait un cliffhanger il faut que le suspense soit associé à une interruption du récit, ce qui ne correspond pas aux cas, beaucoup plus fréquents, d'une simple dilatation de la scène par l'introduction de ce que Roland Barthes appellerait une "catalyse " (1966: 9). Dans ce passage du roman de Thomas Hardy, la catalyse se remarque par un ralentissement stratégique de la scène, par une accumulation de détails qui bloquent la progression de l'intrigue : l'évocation des méditations du protagoniste qui, tout en restant suspendu, se met à réfléchir sur le sens de la vie, sur la géologie, la préhistoire, le darwinisme et les lois de la nature. À l'inverse, dans le cas du cliffhanger, il s'agit d'exploiter un blanc textuel de nature particulière, qui repose sur l'art d'interrompre le récit « au moment où est créée une tension qui appelle une résolution pressante, ou bien au moment précis où l'on aurait voulu connaitre l'issue des événements que l'on vient de lire » (Iser 1976 : 332). Ainsi que le résume Luke Terlaak Poot :

Le cliffhanger fonctionne en s'appuyant sur une configuration particulière de l'histoire et du discours, une configuration que l'on peut définir comme un nonalignement. [...] Chaque fois que nous rencontrons un cliffhanger, nous trouvons une unité du discours (comme un chapitre ou l'épisode d'un feuilleton) qui se termine avant que la portion de l'histoire racontée ait atteint sa résolution. Le cliffhanger est d'abord et avant tout un lieu où le discours narratif s'arrête trop tôt. Par trop tôt, je veux dire que l'événement ou l'épisode raconté n'est pas résolu au moment où le discours arrête. (Terlaak Poot $2016: 52$, m.t.)

3 Au vu de la discontinuité qui caractérise le cliffhanger, cet effet semble ainsi indissociable de l'émergence d'une littérature périodisée, notamment de l'essor du roman-feuilleton à partir du début du XIX siècle, dont Ugo Dionne (2008) a montré l'impact sur l'exploitation fonctionnelle de la discontinuité chapitrale ${ }^{3}$. Pourtant, il est facile de montrer que cette technique de segmentation paroxystique ou, pour reprendre les termes de Terlaak Poot, d'exploitation fonctionnelle d'un non-alignement entre les séquences du récit et de l'histoire, est beaucoup plus ancienne, ainsi qu'en témoignent les récits de Shéhérazade, volontairement suspendus par le lever du soleil, ou le genre des « contesattrapes ", qui tronquent intentionnellement leur dénouement pour frustrer le public ${ }^{4}$.

Il faut ajouter par ailleurs que, s'il y a bien, dans le cas du cliffhanger, une suspension du récit (ou ce que certains critiques définissent comme un suspens), il n'y a pas nécessairement la création d'un effet de suspense. L'utilité de distinguer la technique narrative de l'effet produit sur le public, justifie selon moi l'importance de ne pas céder 
au purisme de certains critiques qui refusent l'usage d'un emprunt de l'anglais et qui considèrent que suspens et suspense sont des termes équivalents. Quand Hitchcock définit le suspense, il le considère du point de vue de l'émotion ressentie par le public, à l'instar de la surprise, qui s'en distingue surtout, selon lui, par la durée du pathos. Pour lui, les émotions apparaissent ainsi comme "un ingrédient nécessaire au suspense " (Truffaut 1975 : 82). À l'inverse, le suspens ou la suspension renvoient plutôt à une technique narrative et non à l'effet esthétique qui lui est associé. Par ailleurs, le suspense n'est pas la seule émotion liée au développement d'une intrigue : Tzvetan Todorov a été l'un des premiers narratologues à souligner la différence de nature entre les effets de curiosité et de suspense; distinction qui sera reprise plus tard, sous diverses appellations, par Sternberg (1978), Phelan (1989) et bien d'autres. Todorov affirme en effet qu'il existe :

deux formes d'intérêt tout à fait différentes. La première peut être appelée la curiosité; sa marche va de l'effet à la cause [...]. La deuxième forme est le suspense et on va ici de la cause à l'effet: on nous montre d'abord les causes, les données initiales [...] et notre intérêt est soutenu par l'attente de ce qui va arriver, c'est-àdire des effets. (Todorov $1971: 60$ )

5 Par conséquent, si la suspension du récit propre au cliffhanger est bien associée à une "tension qui appelle une résolution pressante», ainsi que l'affirme Iser, cette tension peut indifféremment reposer sur des interrogations qui portent sur les causes ou sur les effets d'une situation, et le suspense ne représente donc que de l'une de ses modalités.

\section{La dimension réflexive de la charnière du feuilleton}

Il faut également souligner un autre trait typique des cliffhangers, qui les distingue d'autres formes de dispositifs intrigants, que l'on pourrait qualifier, par contraste, de linéaires ou de discrets. En effet, cette rupture s'accompagne souvent d'une réflexivité plus ou moins marquée portant sur les rouages narratifs qui donnent son élan à l'intrigue, comme si l'on assistait à une sorte de coupe transversale du récit. Le simple effet de pause inhérent à l'interruption du récit, en produisant un effet contre-immersif, facilite la prise de conscience par le lecteur des dispositifs qui orientent son attente du prochain épisode. Mais la narration va souvent plus loin en explicitant les incertitudes de l'histoire ou en formulant les questions auxquels la suite du récit « à suivre » apportera des réponses.

Guy Gauthier (1994) a montré que les aventures de Flash Gordon, publiées en feuilleton à partir de 1934, permettaient d'observer l'établissement progressif de cette technique, qui deviendra canonique à partir des années $1940^{5}$. À l'origine, le dispositif se fonde sur un cliffhanger élémentaire, qui repose sur la représentation d'une situation particulièrement tendue dans la dernière case de la planche. L'image est généralement assortie de la mention « to be continued... » et, éventuellement, d'un récitatif qui souligne le caractère dramatique de l'évémenement montré par l'image. Ce procédé est visible dès le premier épisode de la série, qui se termine par une vignette mettant en scène ce qui apparaît comme une collision imminente entre un vaisseau dans lequel se trouvent embarqués les héros, et une planète folle qui se dirige vers la terre. Le récitatif, en redondance avec le dessin, souligne que la fusée "fonce directement sur une planète menaçante avec un homme fou aux commandes ». On retrouve ce même procédé dans une série française publiée à partir de 1946, ouvertement inspirée par les aventures de Flash Gordon, le 
récitatif expliquant que « jailli de l'infini, un énorme météorite se dirige sur la fusée... une fausse manœuvre et c'est la mort ! À suivre... » :
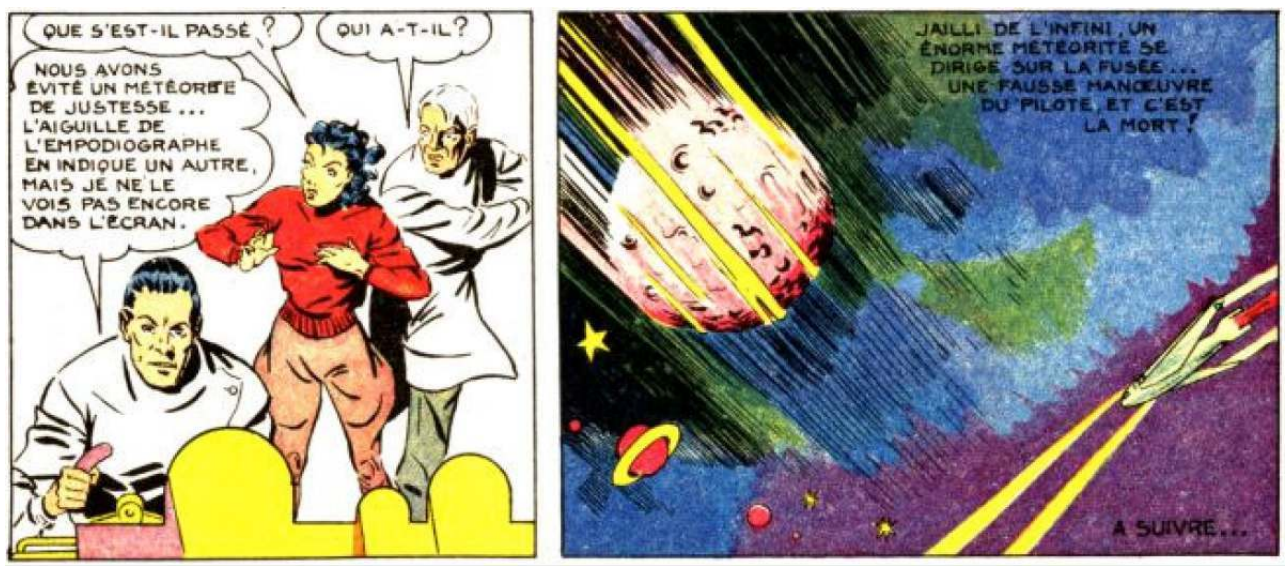

Figure 1 : Kline (Roger Chevallier), Kaza le martien (1946)

Dans le cas présent, on constate que la vignette qui précède la clôture de l'épisode joue également sur l'usage de questions soulignant l'incertitude du récit: "Que s'est-il passé ?» «Qui (sic) a-t-il ?», s'écrient le savant Etienne Launay et sa fille Jane. En l'occurrence, ces questions reçoivent rapidement une réponse par l'intermédiaire de l'assistant du professeur, Jacques, qui explique: «Nous avons évité un météorite de justesse »; mais la curiosité du lecteur est immédiatement repiquée pour étendre son effet à la vignette finale de la planche lorsque le héros ajoute: «l'aiguille de l'empodiographe en indique un autre, mais je ne le vois pas encore sur l'écran ».

Ce jeu entre des interrogations explicites et des réponses diégétiques stratégiquement ajournées, deviendront rapidement un procédé stéréotypé dans le champ de la bande dessinée publiée en feuilleton, les questions pouvant être indifféremment formulées par le récitatif d'un narrateur impersonnel ou par les bulles de dialogues ou de pensées des personnages, voire dans cette catégorie intermédiaire et ambiguë des monologues en aparté qui, à l'instar des récitatifs, semblent en réalité s'adresser aux lecteurs, à l'instar de Tintin dans le Lotus Bleu qui, enlevé et attaché sur une chaise, s'interroge : « Ils m'ont emmené ici et m'ont enfermé dans cette pièce... Que vont-ils faire de moi?». De telles questions s'articulent souvent à un effet de cliffhanger lorsqu'elles surviennent dans la dernière vignette d'un feuilleton, profitant de la clôture provisoire du récit pour repousser la réponse au prochain épisode, comme dans l'exemple suivant, publié en 1947 dans le journal Bayard ${ }^{6}$ : 


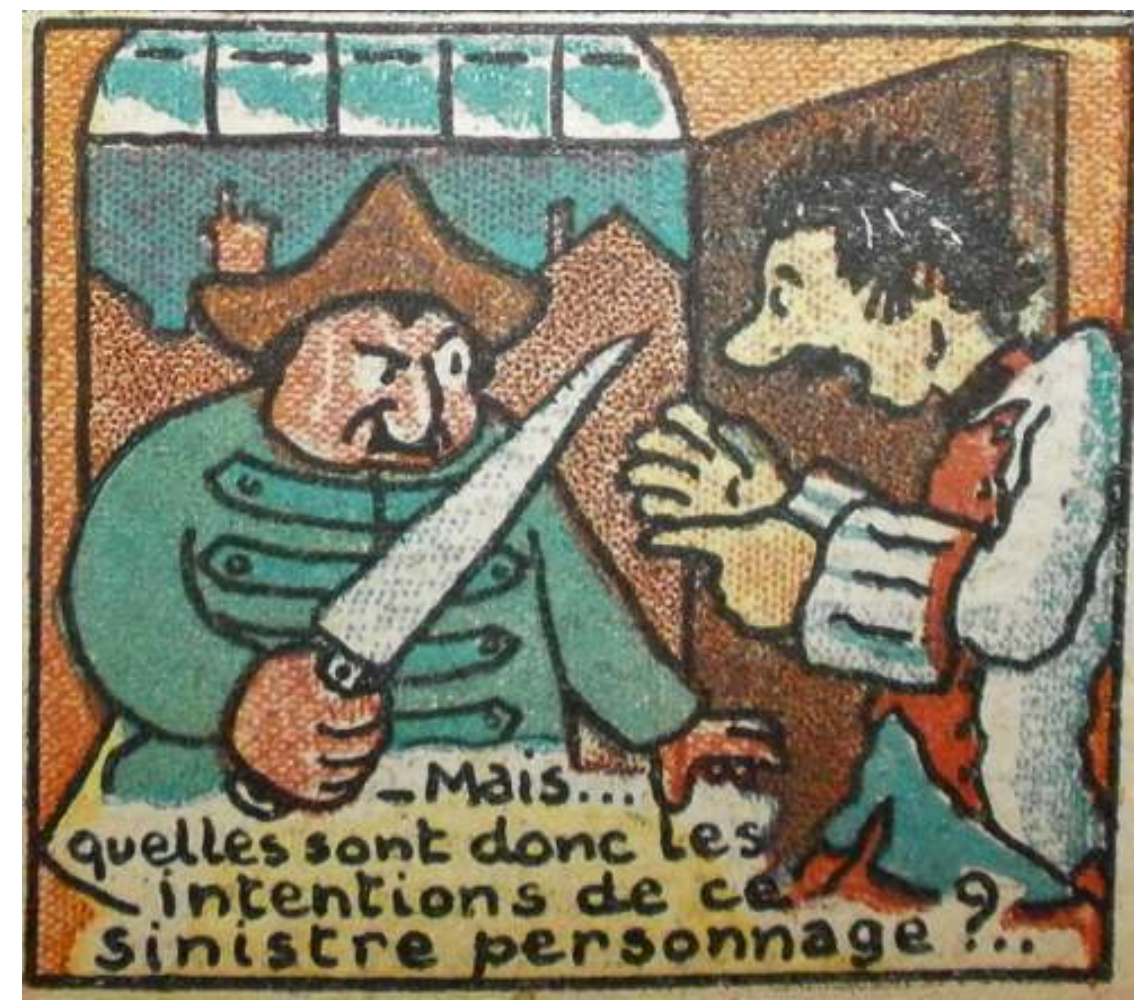

Figure 2 : Bayard (5 janvier 1947) mesure où elle ne s'intègre pas à un dialogue et qu'elle n'appelle pas non plus de réponse directe : le récitatif se contente d'expliciter les questions que devrait se poser un lecteur face à l'irruption soudaine d'un personnage inconnu et menaçant, qui suscite, en l'occurrence autant de curiosité que de suspense, car ses intentions demeurent provisoirement inconnues. Dans la série Flash Gordon, la première occurrence de ces questions explicites en situation de clôture de l'épisode survient dans un contexte où, paradoxalement, la situation narrative ne paraît guère susceptible de créer l'attente d'une suite.

Et maintenant, que vont faire Flash Gordon et Dale?

\section{Zarkov acceptera-t-il l'invitation?}

Retourneront-ils sur la terre ? Ou bien resteront-ils sur la planète $\mathrm{Mongo}^{7}$ ?

Dans un tel cas, il vaudrait mieux parler de pseudo-cliffhanger, car c'est le défaut de tension qui motive le rappel d'incertitudes plus diffuses, en dépit du sentiment de clôture que risquerait éprouver le public. Toutefois, ainsi que l'illustrent les autres exemples que j'ai mentionnés, les questions explicites ne sont pas toujours en décalage avec les véritables enjeux dramatiques du récit et peuvent très bien fonctionner en redondance. Le narrateur ou le personnage qui formule ces questions, à l'instar des parents qui lisent un conte à leur enfant et qui le commentent pour en faciliter la compréhension, remplissent alors une fonction de "régie", en clarifiant la nature des incertitudes qui nouent de l'intrigue. Lorsque ces interrogations se situent à la charnière de l'épisode, elles peuvent également servir d'argument publicitaire, en mettant en relation les incertitudes du récit avec la promesse de leur comblement ultérieur. Cette fonction est particulièrement visible dans une planche qui clôture le deuxième tome de la bande dessinée de Tardi, Le 
Cri $\mathrm{du}$ peuple, qui se termine par la promesse que le lecteur connaitra la réponse aux questions dont le narrateur dresse l'inventaire « en lisant la troisième et dernière partie de cette histoire ${ }^{8} »$.

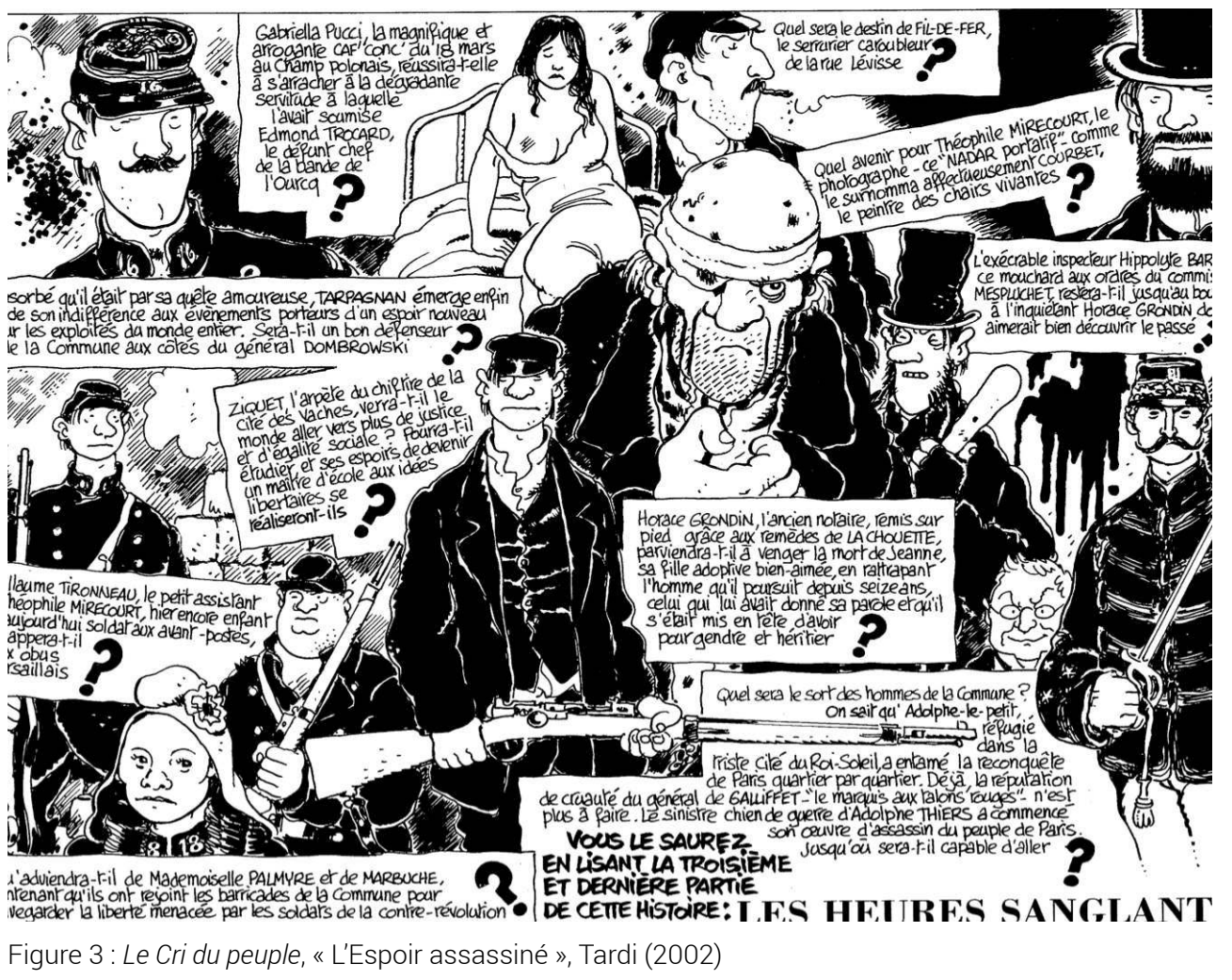

12 Cette planche, dont le ton publicitaire est volontairement sursignifié, pastiche autant les codes de la bande dessinée que ceux du roman-feuilleton. En effet, de tels commentaires se rencontrent fréquemment dans les romans du XIX siècle, sous une forme certes moins standardisée que dans « l'âge d'or » des bandes dessinées. Ainsi, dans Les Mystères de Paris, question et promesse de résolution peuvent être insérés dans des dialogues, comme dans l'exemple suivant, qui se situe à la charnière entre deux chapitres ${ }^{9}$ :

- Et depuis, ni lui ni cette dame ne sont revenus?

— Non ; mais attendez donc la fin de l'histoire, dit Mme Pipelet.

IX. LES TROIS ETAGES

— La fin de l'histoire, la voilà, reprit Mme Pipelet.

(Sue $2009: 209$ )

13 Comme dans les bandes dessinées "à suivre", ce discours associe la curiosité du narrataire à l'injonction du narrateur : «attendez donc la fin de l'histoire ». Narrateur et narrataire imitent ainsi l'interaction de l'auteur avec ses lecteurs, et mettent en évidence une caractéristique essentielle de la dynamique narrative du roman-feuilleton, à savoir qu'il s'agit d'un discours bavard ${ }^{10}$, qui ne cesse de repousser son dénouement en jouant parfois sur la discontinuité d'un récit publié dans un support périodique. Certes, dans la version en volume, la césure intercapitulaire est réduite à un espace occupé par l'intertitre, mais ce «blanc textuel » n'en renvoie pas moins à la césure fondamentale dont dépend la dynamique du cliffhanger. 


\section{Un double attachement au monde et à ses virtualités}

14 d'une réponse diégétique, nous permettent donc d'identifier la fonction publicitaire du cliffhanger, qui passe par la garantie que le manque, mis en évidence par les questions rhétoriques, sera comblé, du moins pour autant que le lecteur consente à acheter le support contenant les développements ultérieurs de l'histoire. Il est donc impossible d'ignorer la dimension proprement commerciale de cet effet qui consiste à refuser de superposer la clôture de l'épisode avec le dénouement de la tension. Dans le romanfeuilleton le découpage du récit exerce une forme de chantage sur le consommateur en le contraignant à acheter le quotidien du lendemain pour connaître la suite de l'histoire. À un stade ultérieur, on peut ajouter que le journal devient un support publicitaire pour le roman qui paraîtra ensuite dans sa version intégrale ${ }^{11}$.

faut cependant ajouter que le pouvoir d'attraction du cliffhanger ne représente qu'une stratégie parmi d'autres pour fidéliser le lecteur d'une fiction. Parmi ces stratégies, on peut mettre en évidence deux aspects complémentaires de la narrativité, largement discutés par les narratologues, qui sont susceptible de fonder l'intérêt d'un récit. Premièrement, il y a l'attachement au monde raconté, qui peut être plus ou moins complexe, familier ou exotique, intime ou dépaysant, auquel il faut associer évidemment l'intérêt que l'on porte aux personnages qui peuplent ce monde, mais aussi le charme déployé par un lieu ou un univers ${ }^{12}$. Deuxièmement, il y a l'intérêt que l'on porte à l'évolution de ce monde ou à l'exploration de ses origines. La double nature de cet attachement, qui joue autant sur les liens noués avec des êtres fictifs que sur une sorte de frustration programmée, est illustrée par ce commentaire de l'auteur de bande dessinée Jean-Christophe Menu :

A posteriori, je crois que ce principe de discontinuité temporelle a été primordial dans mon attachement premier à la bande dessinée. Le journal de Spirou se présentait comme une collection de feuilletons: deux pages d'un récit, la production d'un suspense en fin de deuxième page, et la mention (à suivre). Dans l'attente du numéro de la semaine suivante, l'imagination était en effervescence, l'histoire se poursuivait à travers de multiples hypothèses dans l'imaginaire, bref cet agencement de récits à suivre induisait un processus de désir. Mais les formes courtes (gags en une page) participaient finalement du même type de désir : même sans (à suivre) et avec une fin en bas de la page, il y avait attente et impatience de retrouver ces personnages et cet auteur-là. (Menu $2010: 303$ )

Probablement qu'aucun récit ne peut se passer entièrement de ces deux pôles d'attraction, qui reposent sur la qualité de l'univers diégétique et sur les virtualités liées à son déploiement temporel. Comme l'a montré Marie-Laure Ryan (1991), l'intérêt du récit repose ainsi, pour une part, sur un réseau d'histoires virtuelles qui sont enchâssées au sein d'un monde raconté, mimétiquement consistant, saisi à un état donné de son développement, et c'est précisément ce réseau qui est mis en évidence par les interrogations explicites du cliffhanger et par sa promesse d'une suite au prochain épisode.

Mais ainsi que le souligne Menu, le fait qu'il existe une forme d'attachement au monde fictionnel qui demeure relativement indépendante du développement de l'histoire explique également que la suspension d'un récit publié en feuilleton n'exige pas nécessairement le recours au cliffhanger. On peut aussi désirer retourner vers une fiction 
pour le simple plaisir de retrouver son univers et ses personnages, et la fonction publicitaire sera alors simplement remplie par des indications garantissant la poursuite du récit et facilitant son identification dans le support, à l'instar du visage de Tintin, transformé en logo dans les journaux.

\section{Une coupure révélatrice de la fonction anthropologique du récit mimétique}

Même si elle n'est pas le seul procédé qui nous attache aux récits « mimétiques » - par ce terme, j'entends désigner l'ensemble des formes narratives, factuelles ou fictionnelles, qui visent intentionnellement à intriguer leurs lecteurs et à produire un effet immersif qui les replace dans la perspective temporelle des événements racontés ${ }^{13}$ - la coupure inhérente au cliffhanger n'en est pas moins révélatrice de la fonction profonde de l'intrigue, qui se présente, quand on la considère dans le mouvement de la lecture, comme un réservoir de potentialités narratives, comme une configuration sans cesse changeante, dont le caractère sous-déterminé apparaît essentiel pour garantir le plaisir du lecteur. Ainsi que le résume Hilary Dannenberg :

La lecture du récit est nourrie par deux aspects différents de l'intrigue. Premièrement, il y a la configuration intra-narrative des événements et des personnages, qui se présente comme une matrice de possibilités ontologiquement instable créée par l'intrigue dans son aspect encore non résolu. Celle-ci, en retour, nourrit le désir cognitif du lecteur d'être en possession du second aspect de l'intrigue : la configuration finale réalisée à la clôture du récit, lorsque (du moins c'est ce qu'espère le lecteur) une constellation d'événements cohérente et définitive sera établie. (Dannenberg $2008: 13$, m.t.)

Pourquoi le lecteur cherche-t-il à éprouver ce «désir cognitif »? Pourquoi accepte-t-il d'entrer dans un jeu qui inverse les lois de la communication ordinaires, qui transgresse presque toutes les maximes conversationnelles réglant les échanges et nous condamne à subir la frustration planifiée et intentionnelle d'un narrateur irrémédiablement bavard ${ }^{14}$ ? Il me semble que l'on ne peut apporter de réponses à ces questions que pour autant que l'on accepte de dépasser une interprétation purement commerciale du dispositif. Certes, le cliffhanger fait vendre, mais ce simple constat n'explique pas pourquoi il suscite un tel attrait sur le lecteur.

Pour comprendre ce pouvoir d'attraction, il faut comprendre le sens du jeu et la raison pour laquelle le dispositif textuel place le lecteur dans la perspective limitée d'un personnage ou d'un témoin direct des événements. Ainsi que l'affirme Monika Fludernik, "l'effet esthétique spécifique du récit» consiste en "l'évocation de la conscience humaine mimétiquement motivée et de son expérience (parfois chaotique) d'être dans le monde » (1996: 30, m.t.). Dorrit Cohn constate pour sa part que :

La narration focalisée sur et par un personnage de fiction [...] crée un effet de présence qui donne l'impression qu'elle s'ouvre sur un avenir inconnu. [...] Seule la fiction $^{15}$ est capable de créer l'impression qu'elle présente les événements historiques au moment où ils ont eu lieu, amenant ainsi à la vie la « matière brute, vivante » de l'expérience, sans les distorsions de l'après-coup. (Cohn 2001 : 228-229)

Le récit mimétique se caractérise ainsi par un "chronotope » singulier (pour reprendre un terme bakhtinien), qui privilégie une représentation se situant au ras de l'expérience, au lieu d'adopter une perspective plus distanciée. Un tel point de vue a pour but de permettre au lecteur de vivre une expérience simulée en se (re)plongeant au cœur du 
déroulement d'un événement encore inachevé. Le récit mimétique se situe ainsi aux antipodes de cette distance plus confortable qui permettrait de mieux saisir les enjeux et de mieux comprendre le «sens » de l'histoire. À l'inverse du discours de l'historien ${ }^{16}$, le récit mimétique entraîne la narration dans un bavardage qui évite soigneusement de dévoiler le dénouement des événements avant les dernières lignes. Certes, il peut ainsi remplir un objectif commercial, mais il accomplit en même temps d'autres fonctions anthropologiques. Ainsi que l'affirme Jean-Marie Schaeffer: "la fiction nous donne la possibilité de continuer à enrichir, à remodeler, à réadapter tout au long de notre existence le socle cognitif et affectif originaire grâce auquel nous avons accédé à l'identité personnelle et à notre être-au-monde" (Schaeffer 1999: 327) et elle accomplit cette fonction non par le biais de l'analyse conceptuelle, mais par celui de «l'exemplification modélisante » (Schaeffer 1999 : 47).

Sur un plan non seulement adaptatif, mais également éthique, voire politique, il faut ajouter que la mise en scène d'un monde marqué par une indétermination provisoire ou définitive nous place dans une posture au sein de laquelle une action responsable est envisageable, parce que le futur apparait sous la forme de potentialités encore ouvertes. Dans la récente crise de la dette traversée par la Grèce, nous avons pu observer de frappantes similitudes entre la technique du cliffhanger et la manière dont se structurait un feuilleton médiatique adossé à l'actualité brûlante d'un événement politique au dénouement imprévisible. Dans l'article suivant, nous retrouvons la désignation explicite d'un suspense, l'annonce d'un dénouement qui ne peut être livré immédiatement et des hypothèses contradictoires qui tentent de scruter l'avenir.

Grèce, encore 5 jours de suspense, au moins

Athènes va formuler d'ici jeudi une demande pour un nouveau programme d'aide aux contours encore très flous. Un conseil européen à 28 se réunira dimanche pour y répondre. Où l'avenir de la Grèce sera tranché : dans ou hors de la zone euro.

Deux jours après le triomphe du «non " au référendum grec, le sommet des chefs d'État et de gouvernement de la zone euro convoqué mardi à Bruxelles n'a débouché sur aucune décision majeure. Les plus optimistes, à l'issue du sommet, assuraient qu'un nouvel élan politique avait été enclenché, et qu'un compromis d'ici la fin de semaine restait à portée de main. Les plus inquiets, à l'inverse, notaient qu'une sortie de la Grèce de la zone euro n'était plus un sujet tabou pour aucun des dirigeants présents. (Lamant 2015 : n.p.)

Dans un tel cas, le caractère intrigant du récit n'est pas intentionnel, mais il correspond à une véritable immersion dans le flux de l'actualité, le suspense étant la conséquence directe des contraintes d'écriture d'un récit factuel portant sur un événement provisoirement inachevé. La différence essentielle entre le cliffhanger et la suspension du feuilleton médiatique ne tient, au fond, qu'à ce qui constitue la cause de cette tension irrésolue de l'épisode ${ }^{17}$. Il s'agit en effet de tenir compte, sur un plan rhétorique, du caractère intentionnel ou, au contraire, involontaire de l'interruption sur laquelle se fonde la tension, de manière à distinguer le cliffhanger propre aux récits mimétiques de ses avatars journalistiques non intentionnels ${ }^{18}$. Ainsi que le précise Terlaak Poot:

un cliffhanger est une pause discursive intervenant de telle sorte que la narration d'un événement particulier apparaisse bloquée, et ce blocage est entendu comme intentionnel. Contrairement à une pause accidentelle et d'origine externe au discours (comme une coupure de courant ou un incendie qui se déclare dans une salle de cinéma, qui vous force soudainement à quitter la salle), le cliffhanger est orchestré par un auteur implicite et, par conséquent, il est lié à la manière dont nous appréhendons la conception globale du récit. (Terlaak Poot 2016: 52, m.t.) 
telle différence permet de mieux entrevoir, par contraste, la fonction " mimétique » du cliffhanger en cernant ce qu'il imite, mais aussi, en déterminant ce qui le sépare de l'objet imité. En l'occurrence, lorsqu'un événement dont nous faisons l'expérience induit de la curiosité ou du suspense, le dénouement demeure inaccessible tout simplement parce qu'il appartient à un avenir relativement imprévisible, qui n'est encore écrit par personne. Quand un tel événement est relayé par la presse, le journaliste n'a pas d'autre choix que de produire un récit segmenté, incomplet, qui prend la forme d'un feuilleton médiatique dont le dénouement reste ouvert. Bien que l'on puisse en tirer un argument publicitaire pour augmenter le tirage des journaux, la discontinuité de ce feuilleton médiatique repose sur une résistance objective posée par l'événement (ou du moins, par les sources accessibles concernant cet événement), ce dernier s'opposant au pouvoir d'un journaliste qui se révèle incapable d'en deviner les causes ou les développements ultérieurs, et par conséquent de configurer un récit complet. L'existence d'une telle contrainte extérieure s'exerçant sur un récit émergent, souligne qu'il existe bel et bien des histoires qui nous arrivent avant que nous soyons en mesure de les raconter ou, plus exactement, en même temps que nous les racontons. Par ailleurs, cette histoire émergente possède une dimension politique ou éthique, puisque ce n'est que lorsque nous nous trouvons au cœur d'une histoire que nous pouvons encore en changer le cours, que nous pouvons envisager de produire une action responsable ${ }^{19}$. Si le récit de l'historien est mieux informé que celui du journaliste, en revanche, il est nécessairement en retard sur l'événement, et toute modification du cours de l'histoire racontée, toute action politique relative à cette histoire passée, apparaît dès lors impossible ${ }^{20}$. Bref, ce n'est qu'en tâtonnant que l'on peut véritablement agir ${ }^{21}$.

Les récits mimétiques ${ }^{22}$, dont le cliffhanger révèle la réticence fondamentale à fournir des réponses aux questions que se posent les lecteurs lors de leur progression dans le récit, ne constituent qu'une imitation du caractère inchoatif des histoires dans lesquelles nous sommes intriqués ${ }^{23}$ : il s'agit d'un simulacre qui n'hésite pas à jouer sur un nonalignement intentionnel de l'information, dont le but est de nous donner l'illusion que nous sommes plongés au cœur des événements, ce qui nous permet d'élargir notre champ d'expériences pratiques. Ce faisant, le récit mimétique permet d'acquérir une plus grande plasticité vis-à-vis d'un monde qui manifeste, lui aussi, des résistances incessantes, qui déborde continuellement de nos cadres cognitifs en nouant des conflits, des aventures ou des mystères, tous ces événements qui forment les intrigues de nos vies. Seul le récit mimétique, par ce qui apparaît à première vue comme un bavardage très peu coopératif, est en mesure d'offrir une simulation de l'expérience que nous pouvons faire des histoires qui nous arrivent. Certes, il s'agit là d'un simulacre et d'un moyen de nous attacher à l'univers raconté, mais ce simulacre possède indéniablement une valeur adaptative et éthique.

\section{BIBLIOGRAPHY}

Baroni, Raphaël (2004) « La valeur littéraire du suspense », A Contrario, nº 2 (1), p. 29-43. 
Baroni, Raphaël (2007), La Tension narrative, Paris, Seuil.

Baroni, Raphaël (2009), L'Euvre du temps, Paris, Seuil.

Baroni, Raphaël \& Anaïs Goudmand (à paraître) « De l'épisode au chapitre : la fonction esthétique de la segmentation narrative », in Pratiques et poétiques du chapitre du XIXe au XXIe siècle, A.

Leblond, C. Colin \& T. Conrad (dir.), Rennes, Presses Universitaires de Rennes.

Baroni, Raphaël, Stéphanie Pahud \& Françoise Revaz (2006) « De l'intrigue littéraire à l'intrigue médiatique : le feuilleton Swissmetal », A Contrario, $\mathrm{n}^{\circ} 4$ (2), p. 123-143.

Barthes, Roland (1966), «Introduction à l'analyse structurale des récits », Communications, n 8, p. 1-27.

Barthes, Roland (1970), S/Z, Paris, Seuil.

Brooks, Peter (1984), Reading for the Plot : Design and Intention in Narrative, Cambridge, Harvard University Press.

Cohn, Dorrit (2001), Le propre de la fiction, Paris, Seuil.

Dannenberg, Hilary (2008), Coincidence and Counterfactuality. Plotting Time and Space in Narrative Fiction, Lincoln, University of Nebraska Press.

Dionne, Ugo (2008), La Voie aux chapitres, Paris, Seuil.

Fludernik, Monika (1996), Towards a "Natural" Narratology, London, Routledge.

Gauthier, Guy (1994), « Dans la bande-dessinée », CinémAction, n 71 161-164.

Grice, Paul H. (1979), « Logique et conversation », Communications, $\mathrm{n}^{\circ}$ 30, p. 57-72.

Iser, Wolfgang (1976), L’Acte de lecture. Théorie de l'effet esthétique, Bruxelles, Pierre Mardaga.

Jouve, Vincent (1992), L'Effet-personnage dans le roman, Paris, PUF.

Labrosse, Claude (2000), «L'avènement de la périodicité », in Médias, temporalités et démocratie, A. Vitalis, J.-F. Tétu, M. Palmer and B. Castagna (dir.), Rennes, Editions Apogée, p. 109-123.

Lamant, Ludovic (2015), « Grèce, encore 5 jours de suspense, au moins », Mediapart, mis en ligne le 8 juillet 2015, consulté le 20 juillet 2015, URL : https://www.mediapart.fr/journal/ economie/080715/grece-encore-cinq-jours-de-suspense-au-moins

Lodge, David (2011 [1992]), « Suspense », in The Art of Fiction, London, Vintage, p. 13-16.

Menu, Jean-Christophe (2010), « Feuilleton et improvisation », in La bande dessinée et son double, Paris, L'Association, p. 303-313.

Petitat, André \& Stéphanie Pahud (2004), « L'interaction narrative et ses surprises. Contesattrapes et autres curiosités de la littérature orale », Poétique, nº 138, p. 159-181.

Phelan, James (1989), Reading People, Reading Plots : Character, Progression, and the Interpretation of Narrative, Chicago, University of Chicago Press.

Schapp, Wilhelm (1992), Empêtrés dans des histoires. L'être de l'homme et de la chose, Paris, Editions du Cerf.

Sternberg, Meir (1993 [1978]), Expositional Modes and Temporal Ordering in Fiction, Bloomington \& Indianapolis, Indiana University Press.

Sue, Eugène (2009 [1842-1843]), Les Mystères de Paris, Paris, Gallimard, « Quarto ».

Terlaak Poot, Luke (2016), « On Cliffhangers », Narrative, n² 24 (1), p. 50-67. 
Ryan, Marie-Laure (1991), Possible Worlds, Artificial Intelligence, and Narrative Theory, Bloomington, Indiana University Press.

Todorov, Tzvetan (1971), « Typologie du roman policier », in Poétique de la prose (dir.), Paris, Seuil, p. 55-65.

Truffaut, François (1975), Le Cinéma selon Hitchcock, Paris, Seghers.

Vanni, Michel (2009), L'Adresse du politique. Essai d'approche responsive, Paris, Les Editions du Cerf.

Vanoost, Marie (2013), « Defining Narrative Journalism Through the Concept of Plot », Diegesis, $\mathrm{n}^{\circ}$ $2(2)$, p. $77-97$.

Waldenfels, Bernhard (2009), Topographie de l'étranger, Paris, Van Dieren éditeur.

\section{NOTES}

1. M.t. indique qu'il s'agit de ma traduction.

2. Finalement, le dénouement est à l'image de son hérö̈ne, un mélange d'ingéniosité et de frivolité : Elfridge se déshabille et se sert de ses jupons pour fabriquer une corde permettant de sauver Knight.

3. Ainsi que nous l'avons montré ailleurs (voir Baroni \& Goudmand à paraître), la clôture d'un épisode dans un feuilleton, même aussi définitoire du genre que Les Mystères de Paris, ne correspond pas nécessairement à un cliffhanger.

4. C'est le cas de La petite clé d'or, un conte qui clôturait les recueils des frères Grimm et dont j'analyse la structure dans (Baroni 2007). Sur le genre des «contes-atrapes», voir (Petitat \& Pahud 2004).

5. Pour une discussion plus détaillée du suspense en bande dessinée, je renvoie à Baroni (2007: chapitre 13).

6. Ces cases sont tirées d'un vaste corpus constitué à partir du Fonds Ghebali déposé à la bibliothèque municipale de Lausanne et qui fait l'objet d'une recherche dirigée par Alain Boillat et Françoise Revaz. La dernière case des planches de Hergé, surtout lorsque le mode de publication dominant était encore le feuilleton (notamment dans les trois premières aventures de Tintin), jouent souvent sur un effet semblable, l'étonnement soudain du protagoniste nourrissant la curiosité du lecteur parce que sa source se situe hors du champ de la case, et qu'il faut donc attendre le prochaine épisode (ou tourner la page) pour l'identifier.

7. Cité dans Gauthier (1994:162-163).

8. En réalité, la promesse ne sera pas tenue, car la série comprendra un quatrième volume, l'auteur n'étant pas parvenu à adapter la fin du roman de Vautrin en un seul tome.

9. Ces passages ont été publiés en feuilleton les 13 et 14 septembre 1842. Lors de la publication en feuilleton, le chapitre IX est en réalité le chapitre VIII de la deuxième partie et est intitulé «Les quatre étages ». Je remercie Anaïs Goudmand de me les avoir signalés.

10. Le patronyme de Madame Anastasie Pipelet, concierge à la fois bavarde et indiscrète, pourrait passer pour un clin d'œil, mais il s'agit ici d'une illusion rétrospective, car c'est bien ce personnage imaginé par Eugène Sue qui est à l'origine du nom commun « pipelette ».

11. Sur cette question, voir Baroni (2004).

12. Jouve (1992) appelle cette attraction «l'effet-personne ».

13. Je précise que tous les récits ne sont pas nécessairement mimétiques, car certains, par leur visée informative, se tiennent à une trop grande distance de l'événement raconté pour immerger le lecteur dans le monde construit par la narration et, en conséquence, pour nouer une intrigue. Je pense notamment aux articles de presse adoptant le principe de la "pyramide inversée», 
selon laquelle l'information la plus importante doit être dévoilée en premier. Cette logique « informative » contraste cependant avec les formes d'écriture que l'on désigne par l'étiquette du «journalisme narratif» (ou « literary journalism»), pour lesquelles la qualité « mimétique » du récit importe autant que dans une fiction (cf. Vanoost 2013). Sur l'opposition entre récit intrigant et récit configurant, voir Baroni (2009).

14. Sur les rapports entre les maximes conversationnelles de Grice (1979), la théorie de la pertinence de Sperber et Wilson, et la dynamique de l'intrigue, je renvoie à Baroni (à paraître).

15. Sur ce point, je ne rejoins pas Dorrit Cohn, dans la mesure où, ainsi que je l'ai déjà évoqué, il existe de nombreux récits factuels susceptibles de produire un effet similaire (Vanoost 2013). Je reformulerais donc « seule la fiction » en « seul le récit mimétique ».

16. Voir Baroni (2009 : 45-94).

17. Sur le feuilleton médiatique, je renvoie à Baroni, Pahud et Revaz (2006). Il faudrait ajouter que, dans cet exemple, le récit n'est pas focalisé sur un personnage, ce qui place le lecteur à une certaine distance de l'expérience. Ou du moins, l'expérience en question est celle des témoins collectifs de ce drame historique. Mais le journaliste pourrait se focaliser par exemple sur le dilemme rencontré par Alexis Tsipras. Dans ce cas, la difficulté de se projeter dans le point de vue individuel d'un personnage politique pourrait alors représenter une limite à laquelle le récit de fiction n'est pas contraint.

18. Pour nuancer cette distinction, il faut cependant noter que les coupures publicitaires de certaines émissions de télévision sont souvent placées à des endroits tout aussi stratégiques : par exemple, la diffusion du concours de Miss France est temporairement interrompue juste avant l'annonce de l'heureuse gagnante.

19. Ainsi que l'affirme Claude Labrosse, le journaliste politique est celui qui « tente d'apercevoir le futur sous forme de possibilités argumentées. Le temps n'apparaît pas seulement comme le réservoir organisé des choses accomplies, on peut aussi le percevoir et même le penser comme un horizon raisonnable fait d'actions probables et de décisions réalisables. L'on touche ici à l'ensemble des conceptions modernes du politique » (Labrosse $2000: 120$ ).

20. Il est en revanche évident que le discours historiographique peut avoir une fonction politique lorsqu'il oriente l'histoire actuelle dans laquelle il s'insère, ce qui est tout autre chose.

21. Sur le tâtonnement inhérent à l'action responsable, ma réflexion a été nourrie par les travaux de Bernhard Waldenfels (2009) et, dans son sillage, de Michel Vanni (2009).

22. L'existence du « journalisme narratif » souligne que le genre journalistique peut adopter, au besoin, les procédures du récit mimétique, mais dans ce cas, l'immersion est fondée sur une reconstruction intentionnelle d'une actualité feinte, qui renvoie en réalité à un événement achevé au moment où le journaliste entame l'écriture. Sans entrer dans les détails, je signale que Marie Vanoost (2013) a mis en évidence les fonctions politiques que l'on peut associer à cet usage spécifique de la narrativité mimétique factuelle.

23. Sur cette notion d'intrication dans des histoires, je renvoie aux travaux du philosophe Wilhelm Schapp, ainsi qu'à mon ouvrage L'œuvre du temps (2009).

\section{ABSTRACTS}

Cliffhanger is a narrative technique based on the interruption of a narrative when a tension is created, calling for urgent resolution. It involves a desynchonization of textual closure and of resolution of plot. This paroxysmal suspension can be based on the arousal of suspense or 
curiosity and it is often accompanied by an explicit presentation of unresolved uncertainties, and by metadiegetic comments inviting the receiver to wait for the next developments of the story. The break is usually associated with a periodical publication, but one can find traces of it in chapters of a novel or in the succession of pages in comics previously published in a serialized form. There are also cliffhangers in folktales, as evidenced by the suspenseful stories of Scheherazade in The 1001 Nights. Cliffhanger can be considered as a commercial technique, but it also highlights some of the key features of mimetic narratives, which appear as representations of events rooted in experienciality, where the storyworld appears as an ontologically unstable matrix of virtualities corresponding to plot in its unresolved status. As illustrated by a comparison with serialized informations in news media, this perspective offers a simulation of events involving the ethical or political responsibility of the subject.

Le cliffhanger est une technique narrative qui consiste à introduire une rupture dans le récit au moment où est créée une tension qui appelle une résolution pressante. Il consiste à désynchroniser la clôture textuelle de la résolution de l'intrigue. Cette suspension paroxystique peut reposer sur la création d'un effet de suspense ou de curiosité et il est souvent accompagné d'une explicitation des incertitudes laissées en suspens et de commentaires métadiégétiques invitant le récepteur à attendre la suite du récit. La rupture est généralement associée à un rythme de parution périodique, mais on peut en retrouver des traces atténuées dans le découpage chapitral du roman ou dans la succession des unités paginales d'une bande dessinée. Il existe également des cliffhangers dans certains récits folkloriques, ainsi qu'en témoignent les récits suspendus de Shéhérazade dans Les 1001 Nuits ainsi que le genre des contes-attrapes. Le cliffhanger peut être considéré comme une technique commerciale, mais il met également en lumière certaines caractéristiques essentielles des récits mimétiques, qui apparaissent comme des représentations d'événements ancrées dans l'actualité d'une expérience, au sein de laquelle le monde de l'histoire apparaît comme une matrice de possibilités ontologiquement instable correspondant à l'intrigue dans son état non-résolu. Ainsi que l'éclaire la comparaison avec les feuilletons médiatiques, cette perspective permet d'offrir une simulation d'événements engageant la responsabilité éthique ou politique du sujet.

\section{INDEX}

Chronological index: XXe siècle

Mots-clés: cliffhanger, suspension, suspense, curiosité, tension narrative, feuilleton, mimesis

\section{AUTHOR}

\section{RAPHAËL BARONI*}

Université de Lausanne

Raphaël Baroni est professeur associé à l'Université de Lausanne. Ses travaux portent sur les tensions qui nouent des intrigues dans les récits de fiction. Il travaille aussi sur une approche comparée des formes narratives dans différents médias et sur la polyphonie et les conflits d'interprétation qui en découlent, notamment dans l'œuvre de Michel Houellebecq. Il est l'auteur d'une centaine d'articles et de trois ouvrages : La tension narrative (Seuil, 2007), L'œuvre du temps (Seuil, 2009) et Les rouages de l'intrigue (Slatkine, à paraître). Il a co-dirigé plusieurs ouvrages collectifs, dont Les passions en littérature (Etudes de Lettres $\left.n^{\circ} 295,2014\right)$, Repenser les récits avec les séries télévisées (Télévision $n^{\circ} 7,2016$ ) et Narrative Sequence in Contemporary Narratology (Ohio State 
University Press, 2016). En 2010, il a créé avec Françoise Revaz le Réseau romand de narratologie ( http://www.narratologie.ch/). Il est aussi membre fondateur du Groupe d'étude sur la bande dessinée à l'Université de Lausanne (GrEBD : http://wp.unil.ch/grebd). 